\title{
Force-detected nuclear magnetic resonance: Recent advances and future challenges
}

\author{
M. Poggio ${ }^{1}$ and C. L. Degen ${ }^{2}$ \\ ${ }^{1}$ Department of Physics, University of Basel, Klingelbergstrasse 82, 4056 Basel, Switzerland \\ ${ }^{2}$ Department of Chemistry, Massachusetts Institute of Technology, \\ 77 Massachusetts Avenue, Cambridge, MA 02139, USA
}

(Dated: June 15, 2010)

\begin{abstract}
We review recent efforts to detect small numbers of nuclear spins using magnetic resonance force microscopy. Magnetic resonance force microscopy (MRFM) is a scanning probe technique that relies on the mechanical measurement of the weak magnetic force between a microscopic magnet and the magnetic moments in a sample. Spurred by the recent progress in fabricating ultrasensitive force detectors, MRFM has rapidly improved its capability over the last decade. Today it boasts a spin sensitivity that surpasses conventional, inductive nuclear magnetic resonance detectors by about eight orders of magnitude. In this review we touch on the origins of this technique and focus on its recent application to nanoscale nuclear spin ensembles, in particular on the imaging of nanoscale objects with a three-dimensional (3D) spatial resolution better than $10 \mathrm{~nm}$. We consider the experimental advances driving this work and highlight the underlying physical principles and limitations of the method. Finally, we discuss the challenges that must be met in order to advance the technique towards single nuclear spin sensitivity - and perhaps - to 3D microscopy of molecules with atomic resolution.
\end{abstract}

PACS numbers:

\section{INTRODUCTION}

Advances in the fabrication and measurement of microelectromechanical systems (MEMS) and their evolution into nanoelectromechanical systems (NEMS) have allowed researchers to measure astoundingly small forces, masses, and displacements ${ }^{1-4}$. Ultrasensitive mechanical sensors have been used in a variety of experiments from the measurement of Casimir forces predicted by cavity quantum electrodynamics ${ }^{5}$, to the testing of quantum theories of gravity at nanometer length scales ${ }^{6}$, to the measurement of single electron spins ${ }^{7}$. Recently, micromechanical detectors have provided the clearest picture to date of persistent currents in normal metallic rings ${ }^{8}$. At this stage, the measurement of forces at the attonewton level has been reported by several teams of researchers ${ }^{7,9-12}$. At the same time, mechanical displacement sensitivities are approaching the standard quantum limit, i.e. the fundamental limit to position resolution set by back-action effects ${ }^{12}$. NEMS devices operated at very low temperatures are themselves approaching the quantum regime, with thermal vibration energies only 100 quanta above the zero-point motion of the resonators ${ }^{11,13-15}$. The availability of devices with such exquisite force, mass, and displacement sensitivities has not only allowed for the study of a wide class of condensed matter physics problems, but it has also led to new high resolution nano- and atomic-scale imaging techniques. Magnetic resonance force microscopy, which is reviewed here, has made important contributions to all of these emerging research areas.

Magnetic resonance force microscopy (MRFM) combines the physics of magnetic resonance imaging (MRI) with the techniques of scanning probe microscopy. In MRFM, a nanomechanical cantilever is used to sense the tiny magnetic force arising between the electron or nu- clear spins in the sample and a nearby magnetic particle. The MRFM technique was proposed in the early days of scanning probe microscopy as a method to improve the resolution of MRI to molecular lengthscales ${ }^{16,17}$. The visionary goal of this proposal was to eventually image molecules atom-by-atom, so as to directly map the threedimensional atomic structure of macromolecules ${ }^{18}$. Such a "molecular structure microscope" would have a dramatic impact on modern structural biology, and would be an important tool for many future nanoscale technologies. While this ultimate goal has not been achieved to date, the technique has undergone a remarkable development into one of the most sensitive spin detection methods available to researchers today. Among the important experimental achievements are the detection of a single electronic $\operatorname{spin}^{7}$ and the extension of the spatial resolution of MRI from several micrometers to below ten nanometers ${ }^{19}$.

In this review we discuss the improvements made to the MRFM technique over the last four years, and present an outlook of possible future developments. Section II introduces the basics of MRFM and provides a brief historical overview covering earlier work until about 2006 (for a broader discussion of this work the reader is referred to several reviews, for example Refs. ${ }^{20-26}$ ). Section III primarily focuses on work done by the authors and collaborators while in the MRFM group at the IBM Almaden Research Center. We discuss the recent experimental advances that allowed the measurement sensitivity to reach below 100 nuclear spins. We highlight some of the results enabled by this progress, in particular the imaging of individual virus particles and organic nanolayers, both with three-dimensional (3D) resolutions below $10 \mathrm{~nm}$. We also consider two physical phenomena that become important at these small length-scales: the role of statistical fluctuations in spin polarization and the appearance of fast 
spin relaxation by coupling to mechanical modes. In Section IV we discuss promising future directions aimed at improving the sensitivity of nuclear MRFM: the development of improved magnetic tips, of novel nanomechanical sensors, and of sensitive displacement transducers. We conclude with a comparison to other nanoscale imaging and spin detection techniques in Section V, and an outlook of future applications in Section VI.

\section{BACKGROUND}

\section{A. Principle}

MRI and its older brother, nuclear magnetic resonance (NMR) spectroscopy, rely on measurements of the nuclear magnetic moments present in a sample - magnetic moments arising from atomic nuclei with non-zero nuclear spin. In conventional magnetic resonance detection, the sample is placed in a strong static magnetic field in order to produce a Zeeman splitting between the nuclear spin states. The sample is then exposed to a radio-frequency (rf) magnetic field of a precisely defined frequency. If this frequency matches the Zeeman splitting (which at a given static field is different for every non-zero nuclear spin isotope), then the system absorbs energy from the rf radiation resulting in transitions between the nuclear spin states. From a classical point of view, the total nuclear magnetic moment of the sample starts changing its orientation. Once the rf field is turned off, any component of the total moment remaining perpendicular to the static field is left to precess about this field. The precession of this ensemble of nuclear magnetic moments produces a time-varying magnetic signal that can be detected with a pick-up coil. The electric current induced in the coil is then amplified and converted into a signal that is proportional to the number of nuclear moments (or spins) in the sample. In MRI this signal can be reconstructed into a 3D image of the sample using spatially varying magnetic fields and Fourier transform techniques. The magnetic fields produced by nuclear moments are, however, extremely small: more than one trillion $\left(10^{12}\right)$ nuclear spins are typically needed to generate a detectable signal.

The MRFM technique attempts to improve on the poor detection sensitivity of inductive pick-up coils by mechanically detecting the magnetic forces produced by nuclear moments. To grasp the basic idea behind the method, imagine taking two refrigerator magnets and holding them close together; depending on the magnets' orientation, they exert either an attractive or repulsive force. In MRFM, a compliant cantilever is used to sense the same magnetic forces arising between the nuclear spins in a sample and a nearby nano-magnet. First, either the sample (containing nuclear moments) or the nano-magnet must be fixed to the cantilever. Then, using the techniques of conventional NMR described above, the nuclear spins are made to periodically flip, generating an oscillating magnetic force acting on the cantilever. In order to resonantly excite the cantilever, the nuclear spins must be inverted at the cantilever's mechanical resonance frequency. The cantilever's mechanical oscillations are then measured by an optical interferometer or beam deflection detector. The electronic signal produced by the optical detector is proportional both to the cantilever oscillation amplitude and the number of nuclear spins in the imaging volume. Spatial resolution results from the fact that the nano-magnet produces a magnetic field which is a strong function of position. The magnetic resonance condition and therefore the region where the spins periodically flip is confined to a thin, approximately hemispherical "resonant slice" that extends outward from the nano-magnet (see Figs. 1 and 6). By scanning the sample in 3D through this resonant region, a spatial map of the nuclear spin density can be made.

The advantage of force-detected over inductive techniques is that much smaller devices can be made. In the latter case, the measurement can only be sensitive if the nuclear spins significantly alter the magnetic field within the pick-up coil, i.e. if the spins fill a significant fraction of the coil volume. For spin ensembles with volumes significantly smaller than $(1 \mu \mathrm{m})^{3}$, it is extremely challenging to realize pick-up coils small enough to ensure an adequate filling factor. As a result, even the best resolutions achieved by inductively detected MRI require sample volumes of at least $(3 \mu \mathrm{m})^{327}$. Mechanical resonators, in contrast, can now be fabricated with dimensions far below a micron, such that the sample's mass (which is the equivalent to the filling volume in a pick-up coil) is always significant compared to the bare resonator mass. In addition, mechanical devices usually show resonant quality factors that surpass those of inductive circuits by orders of magnitude, resulting in a much lower baseline noise. For example, state-of-the art cantilever force transducers achieve quality factors between $10^{4}$ and $10^{7}$, enabling the detection of forces of aN/ $\mathrm{Hz}^{1 / 2}$ - less than a billionth of the force needed to break a single chemical bond. In addition, scanning probe microscopy offers the stability to position and image samples with nanometer precision. The combination of these features allows mechanically detected MRI to image at resolutions that are far below one micrometer, and - in principle - to aspire to atomic resolution.

\section{B. Early MRFM}

The use of force-detection techniques in NMR experiments dates back to Evans in $1955^{28}$, and was also used in torque magnetometry measurements by Alzetta and coworkers in the sixties ${ }^{29}$. In 1991 Sidles, independent of this very early work, proposed that magnetic resonance detection and imaging with atomic resolution could be achieved using microfabricated cantilevers and nanoscale ferromagnets. The first micrometer-scale experimental demonstration using cantilevers was realized 

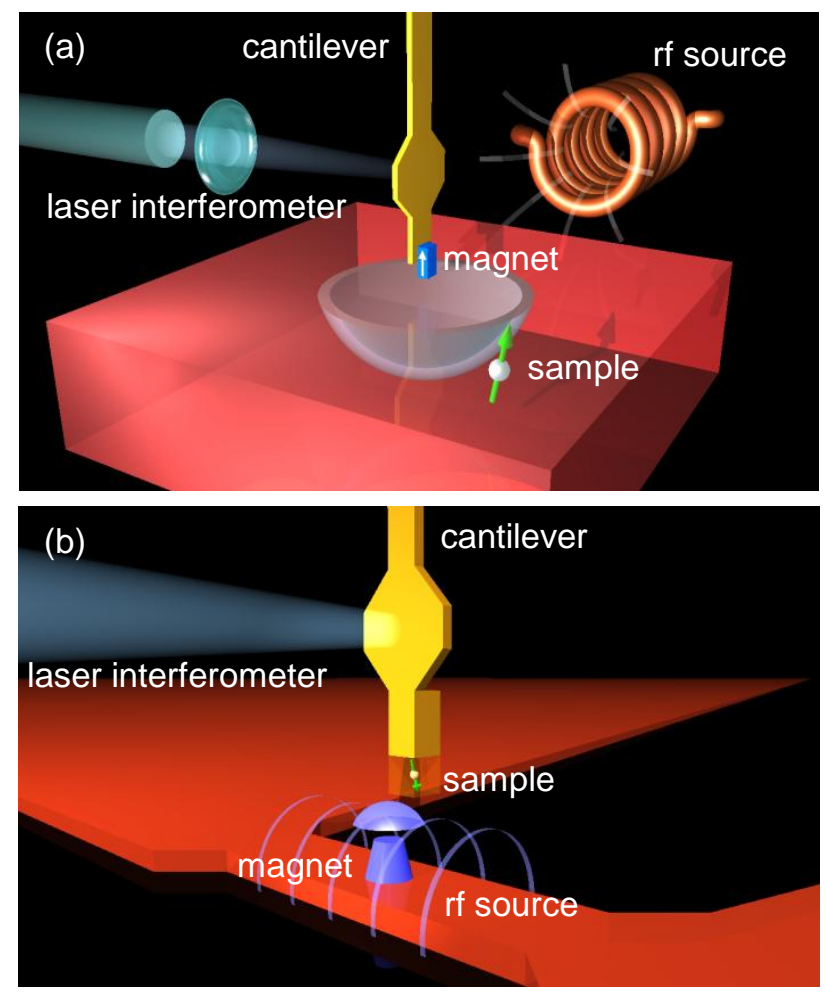

FIG. 1: Schematics of an MRFM apparatus. (a) corresponds to the "tip-on-cantilever" arrangement, such as used in the single electron MRFM experiment of $2004^{7}$. (b) corresponds to the "sampleon-cantilever" arrangement, like the one used for the nanoscale virus imaging experiment in $2009^{19}$. In both cases the hemispherical region around the magnetic tip is the region where the spin resonance condition is met - the so-called "resonant slice".

by Rugar ${ }^{30}$, demonstrating mechanically-detected electron spin resonance in a 30-ng sample of diphenylpicrylhydrazil (DPPH). The original apparatus operated in vacuum and at room temperature with the DPPH sample attached to the cantilever. A mm-sized coil produced an rf magnetic field tuned to the electron spin resonance of the DPPH $(220 \mathrm{MHz})$ with a magnitude of $1 \mathrm{mT}$. By changing the strength of a polarizing magnetic field $(8 \mathrm{mT})$ in time, the electron spin magnetization in the DPPH was modulated. In a magnetic field gradient of $60 \mathrm{~T} / \mathrm{m}$, produced by a nearby $\mathrm{NdFeB}$ permanent magnet, the sample's oscillating magnetization resulted in a time-varying force between the sample and the magnet. This force modulation was converted into mechanical vibration by the compliant cantilever. Displacement oscillations were detected by a fiber-optic interferometer achieving a thermally limited force sensitivity of 3 $\mathrm{fN} / \sqrt{\mathrm{Hz}}$.

During the years following this initial demonstration of cantilever-based magnetic resonance detection, the technique has undergone a series of developments towards higher sensitives that, as of today, is about $10^{7}$ times that of the 1992 experiment (see Fig. 2). In the following, we briefly review the important steps that led to these advances while also touching on the application of

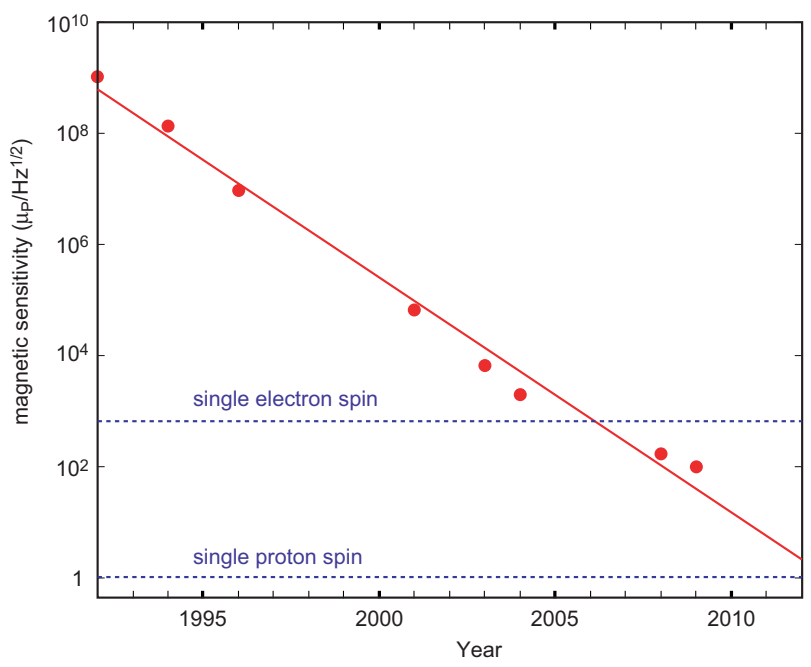

FIG. 2: Advances in the sensitivity of force-detected magnetic resonance over time. Remarkably, improvements have closely followed a "Moore's law" for over a decade, with the magnetic moment sensitivity doubling roughly every eight months. Dots are experimental values $^{7,19,30,31,33,38,60,65}$, and dashed lines indicate sensitivities of one electron and one proton magnetic moment $\left(\mu_{\mathrm{P}}\right)$, respectively.

the technique to imaging and magnetic resonance spectroscopy. Several review articles and book chapters have appeared in the literature that discuss some of these earlier steps more broadly and in richer detail ${ }^{20-23,25,26}$.

Following the initial demonstration of mechanicallydetected electron paramagnetic resonance $(\mathrm{EPR})^{30}$, the MRFM technique was soon extended to NMR in $1994^{31}$ and to ferromagnetic resonance in $1996^{32}$. A major step towards higher sensitivity was made by incorporating the MRFM instrument into a cryogenic apparatus in order to reduce the thermal force noise of the cantilever. A first experiment carried out in 1996 at a temperature of $14 \mathrm{~K}$ achieved a force sensitivity of $80 \mathrm{aN} / \sqrt{\mathrm{Hz}^{33}}$, a roughly 50-fold improvement compared to 1992 mostly due to the higher cantilever mechanical quality factor and the reduced thermal noise achieved at low temperatures. In 1998, researchers introduced the "tip-oncantilever" scheme ${ }^{34}$ (shown in Fig. 1(a)), where the roles of gradient magnet and sample were interchanged. Using this approach, field gradients of up to $2.5 \times 10^{5} \mathrm{~T} / \mathrm{m}$ were obtained by using a magnetized sphere of $3.4-\mu \mathrm{m}$ diameter ${ }^{35}$. These gradients are more than three orders of magnitude larger than those achieved in the first MRFM experiment. In parallel, a series of spin detection protocols were also invented. These protocols include the detection of spin signals in the form of a shift in the cantilever resonance frequency (rather than changes in its oscillation amplitude) ${ }^{36}$, and a scheme that relies on detecting a force-gradient, rather than the force itself ${ }^{37}$. In 2003, researchers approached the level of sensitivity necessary to measure statistical fluctuations in small ensembles of electron spins, a phenomenon that had previously only been observed with long averaging times ${ }^{38}$. Further refinements finally led to the demonstration of single elec- 
tron spin detection in 2004 by the IBM group ${ }^{7}$, which we discuss separately below.

While the bulk of MRFM experiments address the improvement of detection sensitivity and methodology, effort has also been devoted to demonstrate the 3D imaging capacity of the instrument. The first onedimensional MRFM image was made using EPR detection in 1993 and soon after was extended to two and three dimensions ${ }^{39-41}$. These experiments reached about $1-\mu \mathrm{m}$ axial and 5- $\mu \mathrm{m}$ lateral spatial resolution, which is roughly on par with the best conventional EPR microscopy experiments today ${ }^{42}$. In 2003, sub-micrometer resolution (170 $\mathrm{nm}$ in one dimension) was demonstrated with NMR on optically pumped GaAs ${ }^{43}$. In parallel, researchers started applying the technique for the 3D imaging of biological samples, like the liposome, at micrometer resolutions ${ }^{44}$. Shortly thereafter, a $80-\mathrm{nm}$ voxel size was achieved in an EPR experiment that introduced an iterative $3 \mathrm{D}$ image reconstruction technique ${ }^{45}$. The onedimensional imaging resolution of the single electron spin experiment in 2004, finally, was about $25 \mathrm{~nm}^{7}$.

The prospect of applying the MRFM technique to nanoscale spectroscopic analysis has also led to efforts towards combination with pulsed NMR and EPR techniques. MRFM is ill suited to high resolution spectroscopy as broadening of resonance lines by the strong field gradient of the magnetic tip completely dominates any intrinsic spectral features. Nevertheless, a number of advances have been made. In 1997, MRFM experiments carried out on phosphorus-doped silicon were able to observe the hyperfine splitting in the EPR spectrum $^{46}$. Roughly at the same time, a series of basic pulsed magnetic resonance schemes were demonstrated to work well with MRFM, including spin nutation, spin echo, and $T_{1}$ and $T_{1 \rho}$ measurements ${ }^{47,48}$. In 2002, researchers applied nutation spectroscopy to quadupolar nuclei in order to extract local information on the quadrupole interaction ${ }^{49}$. This work was followed by a line of experiments that demonstrated various forms of NMR spectroscopy and contrast, invoking dipolar couplings ${ }^{50}$, cross polarization ${ }^{51,52}$, chemical shifts ${ }^{53}$, and multi-dimensional spectroscopy ${ }^{53}$. Some interesting variants of MRFM that operate in homogeneous magnetic fields were also explored. These techniques include measurement of torque rather than force $\mathrm{e}^{29,54}$ and the socalled "Boomerang" experiment ${ }^{55,56}$.

Finally, while not within the scope of this review, it is worth mentioning that MRFM has also been successfully applied to a number of ferromagnetic resonances studies, in particular for probing the resonance structure of micron-sized magnetic disks. ${ }^{57,58}$.

\section{Single electron MRFM}

The measurement of a single electron spin by the IBM group in 2004 concluded a decade of development on the MRFM technique and stands out as one of the first single-

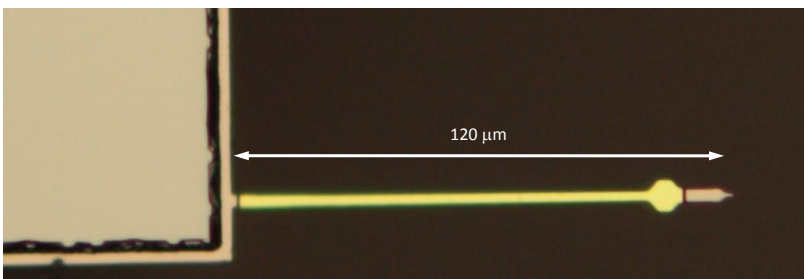

FIG. 3: Image of an ultrasensitive mass-loaded Si cantilever taken from an optical microscope. This type of cantilever, which is about $100-n m$-thick and has a spring constant under $100 \mu \mathrm{N} / \mathrm{m}$, has been used as a force transducer in the many of the latest MRFM experiments $^{59}$.

spin measurements in solid-state physics. A variety of developments led to the exceptional measurement sensitivity required for single-spin detection. These include the operation of the apparatus at cryogenic temperatures and high vacuum, the ion-beam-milling of magnetic tips in order to produce large gradients, and the fabrication of mass-loaded attonewton-sensitive cantilevers ${ }^{59}$ (shown in Fig. 3). The thermal noise in higher order vibrational modes of mass-loaded cantilevers is suppressed compared with the noise in the higher order modes of conventional, "flat" cantilevers. Since high frequency vibrational noise in combination with a magnetic field gradient can disturb the electron spin, the mass-loaded levers proved to be a crucial advance for single-electron MRFM. In addition, the IBM group developed a sensitive interferometer employing only a few nanowatts of optical power for the detection of cantilever displacement ${ }^{10}$. This low incident laser power is crucial for achieving low cantilever temperatures and thus minimizing the effects of thermal force noise. A low-background measurement protocol called OSCAR based on the NMR technique of adiabatic rapid passage was also employed ${ }^{60}$. Finally, the experiment required the construction of an extremely stable measurement system capable of continuously measuring for several days in an experiment whose single-shot signalto-noise ratio was just $0.06^{7}$.

The path to this experimental milestone led through a variety of interesting physics experiments. In 2003, for example, researchers reported on the detection and manipulation of small ensembles of electron spins - ensembles so small that the their statistical fluctuations dominate the polarization signal ${ }^{38}$. The approach developed for measuring statistical polarizations provided a potential solution to one of the fundamental challenges of performing magnetic resonance experiments on small numbers of spins. In 2005, Budakian and coworkers took these concepts one step further by actively modifying the statistics of the naturally occurring fluctuations of spin polarization $^{61}$. In one experiment, the researchers polarized the spin system by selectively capturing the transient spin order. In a second experiment, they demonstrated that spin fluctuations can be rectified through the application of real-time feedback to the entire spin ensemble. 


\section{RECENT STRIDES IN NUCLEAR MRFM}

In the following, we summarize the latest advances made to nuclear spin detection by MRFM. The shift of focus from electron to nuclear spins is driven by the prospect of applying the technique for high-resolution magnetic resonance microscopy. MRI has had a revolutionary impact on the field of non-invasive medical screening, and is finding an increased number of applications in materials science and biology. The realization of MRI with nanometer or sub-nanometer resolution may have a similar impact, for example, in the field of structural biology. Using such a technique, it may be possible to image complex biological structures, even down to the scale of individual molecules, revealing features not elucidated by other methods.

The detection of a single nuclear spin, however, is far more challenging than that of single electron spin. This is because the magnetic moment of a nucleus is much smaller: a hydrogen nucleus (proton), for example, possess a magnetic moment that is only $\sim 1 / 650$ of an electron spin moment. Other important nuclei, like ${ }^{13} \mathrm{C}$ or a variety of isotopes present in semiconductors, have even weaker magnetic moments. In order to observe single nuclear spins, it is necessary to improve the state-of-the-art sensitivity by another two to three orders of magnitude. While not out of the question, this is a daunting task that requires significant advances to all aspects of the MRFM technique. In the following, we discuss some steps made in this direction since 2005. Our focus is on the work contributed by the authors while at the IBM Almaden Research Center. There the authors were part of a team led by Dan Rugar, who has pioneered many of the important developments in MRFM since its experimental beginnings in 1992 .

\section{A. Improvements to microfabricated components}

Improvements in the sensitivity and resolution of mechanically detected MRI hinge on a simple signal-to-noise ratio, which is given by the ratio of the magnetic force power exerted on the cantilever over the force noise power of the cantilever device. For small volumes of spins, we measure statistical spin polarizations, therefore we are interested in force powers (or variances) rather than force amplitudes:

$$
\mathrm{SNR}=N \frac{\left(\mu_{N} G\right)^{2}}{S_{\mathrm{F}} B}
$$

Here, $N$ is the number of spins in the detection volume, $\mu_{N}$ is the magnetic moment of the nucleus of interest, $G$ is the magnetic field gradient at the position of the sample, $S_{F}$ is the force noise spectral density set by the fluctuations of the cantilever sensor, and $B$ is the bandwidth of the measurement, determined by the nuclear spin relaxation rate $1 / \tau_{m}$. This expression gives the single-shot

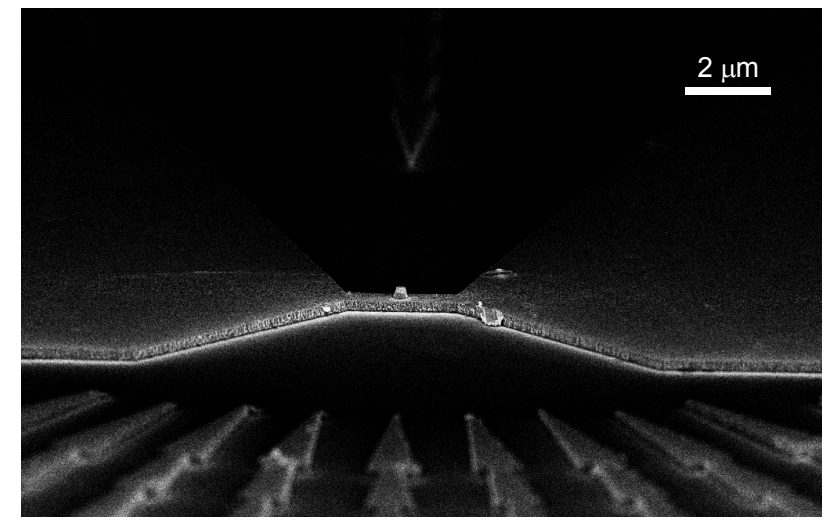

FIG. 4: An SEM image of a $\mathrm{Cu}$ "microwire" $\mathrm{rf}$ source with integrated FeCo tip for MRFM ${ }^{63}$. The arrow-like structures at the bottom of the image provide guidance for aligning the microwire with the cantilever.

signal-to-noise ratio of a thermally-limited MRFM apparatus. The larger this signal-to-noise ratio is, the better the spin sensitivity will be.

From the four parameters appearing in (1), only two can be controlled and possibly improved. On the one hand, the magnetic field gradient $G$ can be enhanced by using higher quality magnetic tips and by bringing the sample closer to these tips. On the other hand, the force noise spectral density $S_{F}$ can be reduced by going to lower temperatures and by making intrinsically more sensitive mechanical transducers.

The latest improvements to MRFM sensitivity rely on advances made to both of these critical parameters. In 2006, the IBM group introduced a micromachined array of Si cones as a template and deposited a multilayer $\mathrm{Fe} / \mathrm{CoFe} / \mathrm{Ru}$ film to fabricate nanoscale magnetic tips ${ }^{62}$. The micromachined tips produce magnetic field gradients in excess of $10^{6} \mathrm{~T} / \mathrm{m}$ owing to their sharpness (the tip radius is less than $50 \mathrm{~nm}$ ). Previously, maximum gradients of $2 \times 10^{5} \mathrm{~T} / \mathrm{m}$ had been achieved by ion-beam-milling SmCo particles down to $150 \mathrm{~nm}$ in size. The gradients from the new nanoscale tips proved to be strong enough to push the resolution of MRI to below $100 \mathrm{~nm}$, in an experiment that is further discussed in the next section.

In the two following years, the group made further improvements to their measurement sensitivity through the development of a magnetic tip integrated onto an efficient "microwire" rf source ${ }^{63}$, illustrated in Fig. 4. This change in the apparatus solved a simple but significant problem: the typical solenoid coils used to to generate the strong rf pulses for spin manipulation dissipate large amounts of power, which even for very small microcoils with a diameter of $300 \mu \mathrm{m}$ amounts to over $0.2 \mathrm{~W}$. This large amount of heat is far greater than the cooling power of available dilution refrigerators. As a result, nuclear spin MRFM experiments had to be performed at elevated temperatures ( $4 \mathrm{~K}$ or higher), thereby degrading the SNR. In some cases the effects can be mitigated through pulse protocols with reduced duty cycles $^{37,62}$, but it is desir- 
able to avoid the heating issue altogether.

Micro-striplines, on the other hand, can be made with sub-micrometer dimensions using e-beam lithography techniques. Due to the small size, the stripline confines the rf field to a much smaller volume and causes minimal heat dissipation. Using e-beam lithography and lift-off, the IBM group fabricated a $\mathrm{Cu}$ "microwire" device that was $0.2 \mu \mathrm{m}$ thick, $2.6 \mu \mathrm{m}$ long, and $1.0 \mu \mathrm{m}$ wide. A stencil-based process was then used to deposit a 200-nm-diameter FeCo tip on top of the wire to provide a static magnetic field gradient. Since the sample could be placed within $100 \mathrm{~nm}$ of the microwire and magnetic tip, rf magnetic fields of over $4 \mathrm{mT}$ could be generated at $115 \mathrm{MHz}$ with less than $350 \mu \mathrm{W}$ of dissipated power. As a result, the cantilever temperature during continuous rf irradiation could be stabilized below $1 \mathrm{~K}$, limited by other experimental factors and not the rf device. Simultaneously, the cylindrical geometry of the magnetic tip optimized the lateral field gradient as compared to the micromachined thin-film Si tips, resulting in values exceeding $4 \times 10^{6} \mathrm{~T} / \mathrm{m}$. As an added benefit, the alignment of the apparatus was simplified as the magnetic tip and the rf source were integrated on a single chip. The cantilever carrying the sample simply needed to be positioned directly above the microwire device. Previous experiments had required an involved three part alignment of magnetic-tipped cantilever, sample, and rf source.

\section{B. MRI with resolution better than $100 \mathrm{~nm}$}

The above instrumental advances to the technique led to two significant experiments that finally demonstrated MRFM imaging resolutions in the low nano-scale. In a first experiment in 2007, Mamin and coworkers used a "sample-on-cantilever" geometry with a patterned 100nm-thick $\mathrm{CaF}_{2}$ film as their sample and a micromachined Si tip array coated with a thin magnetic layer as their magnetic tip ${ }^{62}$. The $\mathrm{CaF}_{2}$ films were thermally evaporated onto the end of the cantilever and then patterned using a focused ion beam, creating features with dimensions between 50 and $300 \mathrm{~nm}$. The cantilevers used in these measurements were custom-made single-crystal Si cantilevers with a $60 \mu \mathrm{N} / \mathrm{m}$ spring constant $^{59}$.

Fig. 5 shows the result of such an imaging experiment, measuring the ${ }^{19} \mathrm{~F}$ nuclei in the $\mathrm{CaF}_{2}$ sample. The resultant image reproduced the morphology of the $\mathrm{CaF}_{2}$ sample, which consisted of several islands of material, roughly 200 -nm-wide and 80 -nm-thick, at a lateral resolution of $90 \mathrm{~nm}$. At a temperature of $600 \mathrm{mK}$ and after 10 minutes of averaging, the achieved detection sensitivity (SNR of one) corresponded to the magnetization of about $1200{ }^{19} \mathrm{~F}$ nuclear moments.
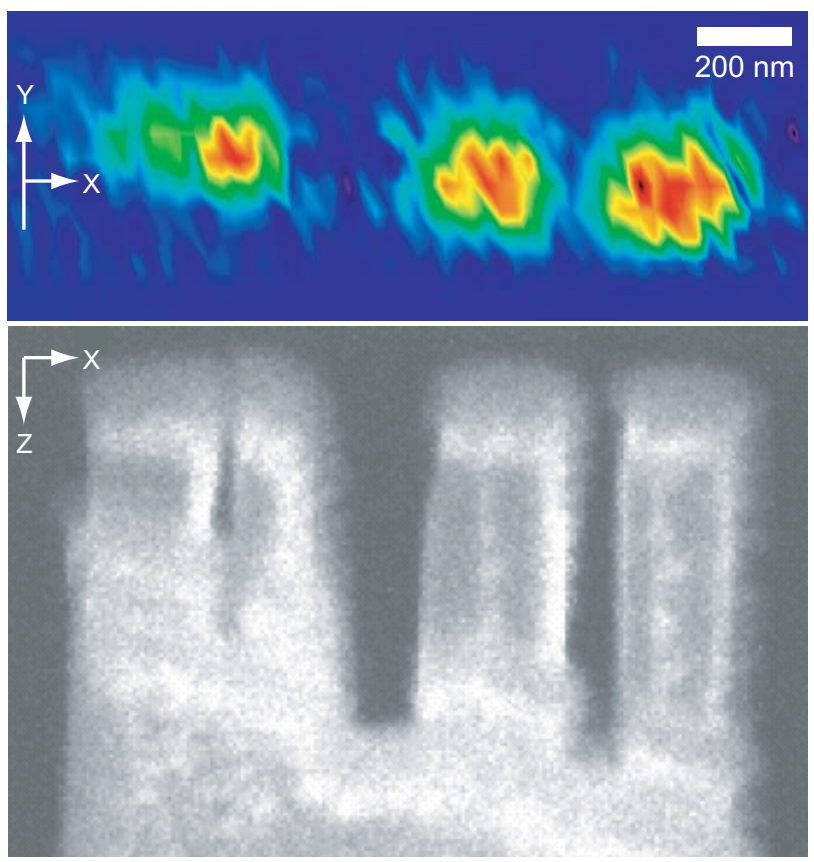

FIG. 5: (a) Two-dimensional MRFM image of ${ }^{19} \mathrm{~F}$ nuclear spins in a patterned $\mathrm{CaF}_{2}$ sample, and (b) corrsponding SEM micrograph (side view) of the cantilever end with the $80 \mathrm{~nm}$ thin $\mathrm{CaF}_{2}$ film at the top of the image. Figure adapted from Ref. ${ }^{62}$.

\section{Nanoscale MRI of virus particles}

Following the introduction of the integrated microwire and tip device, the IBM researchers were able to improve imaging resolutions to well below $10 \mathrm{~nm}^{19}$. These experiments, which used single tobacco mosaic virus (TMV) particles as the sample, both show the feasibility for MRI imaging with nanometer resolution, and the applicability of MRFM to biologically relevant samples.

Fig. 6 is a representation of the MRFM apparatus used in these experiments. The virus particles were transferred to the cantilever end by dipping the tip of the cantilever into a droplet of aqueous solution containing suspended TMV. As a result, some TMV were attached to the gold layer previously deposited on the cantilever end, The density of TMV on the gold layer was low enough that individual particles could be isolated. Then the cantilever was mounted into the low-temperature, ultrahigh-vacuum measurement system and aligned over the microwire.

After applying a static magnetic field of about $3 \mathrm{~T}$, resonant rf pulses were applied to the microwire source in order to flip the ${ }^{1} \mathrm{H}$ nuclear spins at the cantilever's mechanical resonance. Finally, the end of the cantilever was mechanically scanned in three dimensions over the magnetic tip. Given the extended geometry of the region in which the resonant condition is met, i.e. the "resonant slice", a spatial scan does not directly produce a map of the ${ }^{1} \mathrm{H}$ distribution in the sample. Instead, each data point in the scan contains force signal from ${ }^{1} \mathrm{H}$ spins at 


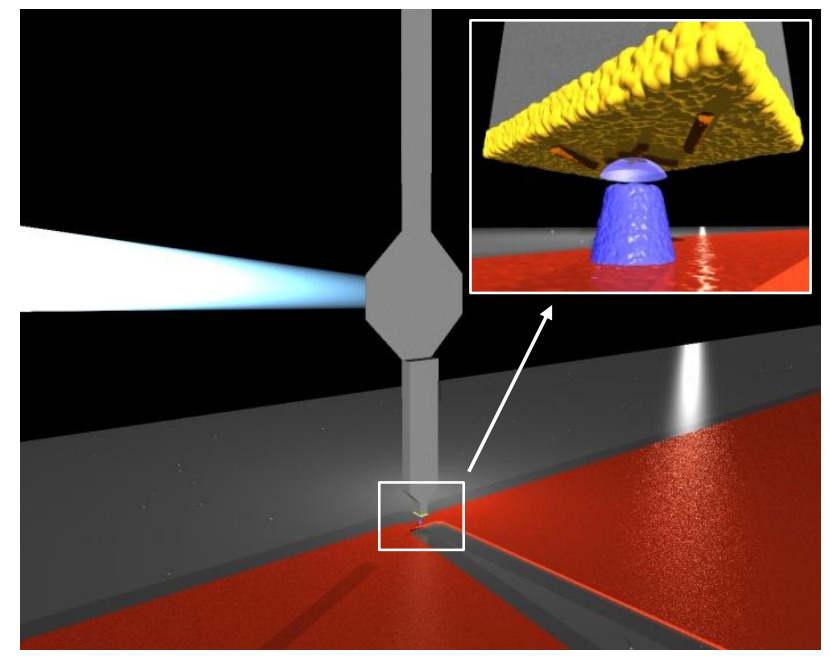

FIG. 6: Artistic view of the MRFM apparatus used for MRI of individual tobacco mosaic virus particles. Pictured in the center is the cantilever, coming from the left is the laser beam used for position sensing, and in red is the $\mathrm{Cu}$ microwire $\mathrm{rf}$ source. The inset shows a close-up representation of the gold-coated end of the cantilever with attached virus particles. On top of the microwire, in blue, is the magnetic FeCo tip with the "mushroom" shaped resonant slice hovering above.

a variety of different positions. In order to reconstruct the three-dimensional spin density (the MRI image), the force map must be deconvolved by the point spread function defined by the resonant slice. Fortunately, this point spread function can be accurately determined using a magnetostatic model based on the physical geometry of the magnetic tip and the tip magnetization. Deconvolution of the force map into the three-dimensional ${ }^{1} \mathrm{H}$ spin density can be done in several different ways; for the results presented in ${ }^{19}$ the authors applied the iterative Landweber deconvolution procedure suggested in an earlier MRFM experiment ${ }^{45,64}$. This iterative algorithm starts with an initial estimate for the spin density of the object and then improves the estimate successively by minimizing the difference between the measured and predicted spin signal maps. The iterations proceed until the residual error becomes comparable with the measurement noise.

The result of a representative experiment is shown in Fig. 7. Here, clear features of individual TMV particles, which are cylindrical, roughly 300-nm-long, and $18 \mathrm{~nm}$ in diameter, are visible and can be confirmed against a scanning electron micrograph (SEM) of the same region (Fig. 8). As is often the case, both whole virus particles and particle fragments are observed. Note that the origin of contrast in MRFM image and the SEM image is very different: the MRFM reconstruction is elementally specific and shows the 3D distribution of hydrogen in the sample; contrast in the SEM image is mainly due to the virus blocking secondary electrons emitted from the underlying gold-coated cantilever surface. In fact, the SEM image had to be taken after the MRFM image

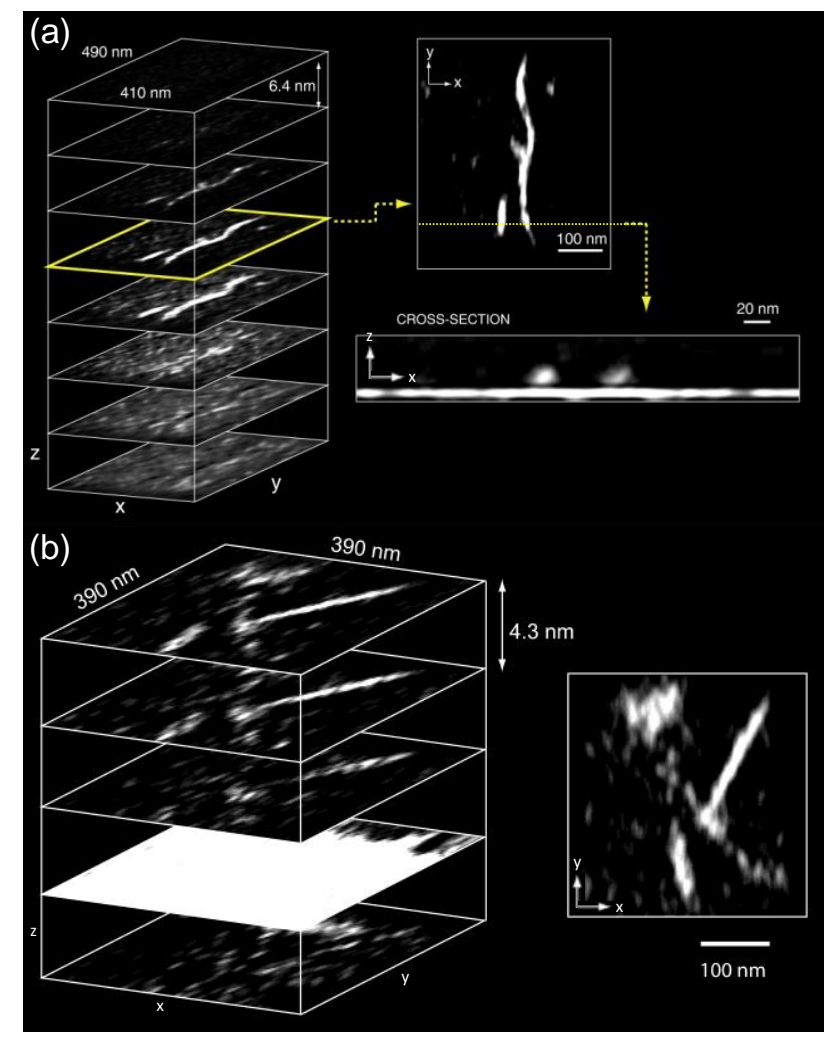

FIG. 7: Nanoscale MRI images of tobacco mosaic virus (TMV) particles acquired by MRFM. (a) The series of images to the left depicts the $3 \mathrm{D}{ }^{1} \mathrm{H}$ spin density of virus particles deposited on the end of the cantilever. Black represents very low or zero density of hydrogen, while white is high hydrogen density. The right side shows a representative xy-plane, with several viral fragments visible, and a cross-section (xz-plane) of two virus particles that reveals an underlying molecular layer of hydrocarbons covering the cantilever surface. (b) $3 \mathrm{D}{ }^{1} \mathrm{H}$ spin density recorded on a different region of the same cantilever as in (a), showing an intact and several fragmented virus particles. The right side shows a representative xy-plane.

as exposure to the electron beam destroys the virus particles. The imaging resolution, while not fine enough to discern any internal structure of the virus particles, constitutes a 1000-fold improvement over conventional MRI, and a corresponding improvement of volume sensitivity by about 100 million.

\section{Imaging organic nanolayers}

In addition to "seeing" individual viruses, the researchers also detected an underlying proton-rich layer. This signal originated from a naturally occurring, subnanometer thick layer of adsorbed water and/or hydrocarbon contamination.

The hydrogen-containing adsorbates picked up on a freshly cleaned gold surface turn out to be enough to produce a distinguishable and characteristic signal. From analysis of the signal magnitude and magnetic field dependence, the scientists were able to determine that the 


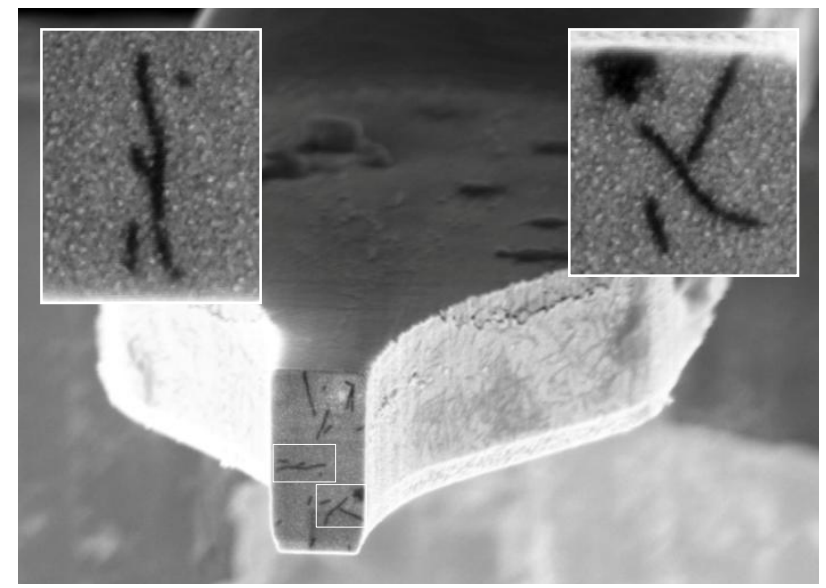

FIG. 8: SEM of the TMV particles and particle fragments on the gold-coated cantilever end. The insets enlarge the areas that were imaged by MRFM in Fig. 7.

adsorbates form a uniform layer on the gold surface with a thickness of roughly 5 to $10 \AA^{65}$.

Using a similar approach, the researchers made a 3D image of a multiwalled nanotube roughly $10 \mathrm{~nm}$ in diameter, depicted in Fig. 9. The nanotube, attached to the end of a 100-nm-thick Si cantilever, protruded a few hundred nanometers from the end of the cantilever. As had been previously observed with gold layers, the nanotube was covered by a naturally occurring protoncontaining contamination layer. Though the magnitude of the signal was roughly 10 times less than that of the two-dimensional layer - reflecting its relatively small volume - it was accompanied by a very low background noise level that made it possible to produce a clear image of the morphology of the nanotube. Using the same iterative deconvolution scheme developed to reconstruct the image of the TMV particles, the researchers produced an image of a cylindrical object, $10 \mathrm{~nm}$ in diameter at the distal end. No evidence was found for the hollow structure that might be expected in the image of such a layer. The experiment did not show any evidence for an empty cylindrical region within the nanotube. Given the small inner diameter (less than $10 \mathrm{~nm}$ ), however, it was not clear whether hydrogen-containing material was in fact incorporated inside the nanotube, or if the resolution of the image was simply not sufficient to resolve the feature.

\section{E. Observation and manipulation of statistical polarizations}

While predominantly driven by the interest in high resolution MRI microscopy, the exquisite spin sensitivity of MRFM also gives us a window into the spin dynamics of small ensembles of spins. When probing nuclear spins on the nanometer scale, for example, random fluctuations of the spin polarization will typically exceed the mean
Boltzmann polarization if sample volumes are smaller than about $(100 \mathrm{~nm})^{3}$, as shown in Fig. 10. This statistical polarization arises from the incomplete cancellation of randomly oriented spins. For an ensemble of $N$ nuclei of spin $1 / 2$ and in the limit of small mean polarization, which is representative of most experiments, the variance of the fluctuations is $\sigma_{\Delta N}^{2} \simeq N$. The existence of statistical polarization was pointed out by Bloch in his seminal paper on nuclear induction ${ }^{66}$, and has been observed experimentally by a number of techniques, including superconducting quantum interference devices ${ }^{67}$, conventional magnetic resonance detection ${ }^{68-70}$, optical techniques $^{71}$, and $\mathrm{MRFM}^{62,72}$.

In a result that was enabled by the latest advances in MRFM detection sensitivity, the IBM scientists were able - for the first time - to follow the fluctuations of a statistical polarization of nuclear spins in real time. These experiments followed the dynamics of an ensemble of roughly $2 \times 10^{6}{ }^{19} \mathrm{~F}$ spins in $\mathrm{CaF}_{2}{ }^{73}$. The challenge of measuring statistical fluctuations presents a major obstacle to nanoscale imaging experiments. In particular, the statistical polarization has random sign and a fluctuating magnitude, making it hard to average signals. An efficient strategy for imaging spin fluctuations is therefore to use polarization variance, rather than the polarization itself, as the image signal. This has recently been demonstrated both by force-detected ${ }^{19,62,65,73}$ and conventional $^{70}$ MRI. Furthermore, it was demonstrated that for cases where spin lifetimes are long, rapid randomization of the spins by rf pulses can considerably enhance the signal-to-noise ratio of the image ${ }^{73}$. In the end, for the purposes of imaging, it is not necessary to follow the sign of the spin polarization; it is enough to simply determine from the measured spin noise where and how many spins are present at a particular location.

The nuclear spin lifetime itself, which is apparent as the correlation time of the nuclear fluctuations $\tau_{m}$, was also shown to be an important source of information. Using suitable rf pulses, researchers demonstrated that Rabi nutations, rotating-frame relaxation times, and nuclear cross-polarization can be encoded in $\tau_{m}$ leading to new forms of image contrast ${ }^{63,74}$.
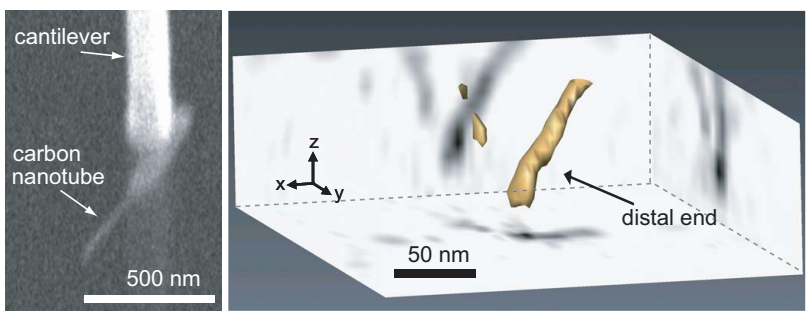

FIG. 9: Scanning electron microscopy image of a 10-nm-diameter carbon nanotube attached to the end of a Si cantilever (left), and MRFM image of the proton density at the nanotube's distal end (right). Figure adapted from Ref. ${ }^{65}$. 


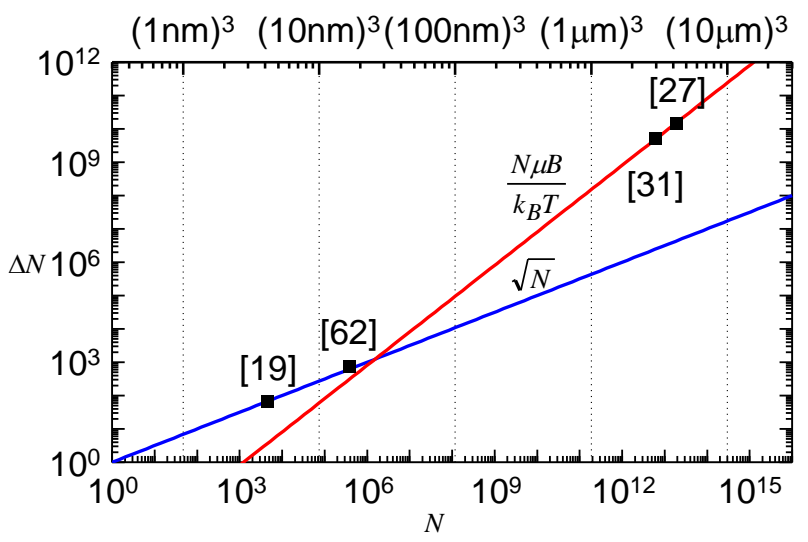

FIG. 10: Comparison of mean thermal (or Boltzmann) polarization $\Delta \bar{D}=N \mu B / k_{\mathrm{B}} T$ versus statistical polarization $\Delta N_{\mathrm{rms}}=\sqrt{N}$ as a function of the number $N$ of nuclear spins in the ensemble. While statistical polarization fluctuations are negligible for macroscopic samples where $N$ is large, they dominate over thermal polarization for small $N$. Under the conditions typical for MRFM, where $T=4 \mathrm{~K}$ and $B=3 \mathrm{~T}$ (shown here), this crossover occurs around $N \approx 10^{6}$, or for sample volumes below about (100 $\mathrm{nm})^{3}$. Dots represent experimental values for conventional $\mathrm{MRI}^{27}$ and MRFM $^{19,31,62}$.

\section{F. Mechanically induced spin relaxation}

The high sensitivity of MRFM is enabled in part by the strong coupling that can be achieved between spins and the cantilever. This coupling is mediated by field gradients that can exceed $5 \times 10^{6} \mathrm{~T} / \mathrm{m}$. The strong interaction between spins and sensor has been the subject of a number of theoretical studies, and is predicted to lead to a host of intriguing effects. These range from shortening of spin lifetimes by "back action" ${ }^{75,76}$, to spin alignment by specific mechanical modes either at the Larmor frequency or in the rotating frame 77,78 , to resonant amplification of mechanical oscillations ${ }^{79}$, to long-range mediation of spin couplings using charged resonator arrays ${ }^{80}$.

Recently the IBM group reported the first direct experimental evidence for accelerated nuclear spin relaxation induced by a single, low-frequency mechanical mode ${ }^{81}$. In these experiments the slight thermal vibration of the cantilever generated enough magnetic noise to destabilize the spin. Enhanced relaxation was found when one of the cantilever's upper modes (in particular the third mode with a frequency of about $120 \mathrm{kHz}$ ) coincided with the Rabi frequency of the spins. In this "strong coupling" regime, the cantilever is more tightly coupled to one mechanical resonator mode than to the continuum of phonons that are normally responsible for spin-lattice relaxtation. Interestingly, these initial experiments showed a scaling behavior of the spin relaxation rate with important parameters, including magnetic field gradient and temperature, that is substantially smaller than predicted by theory (see Fig. 11).

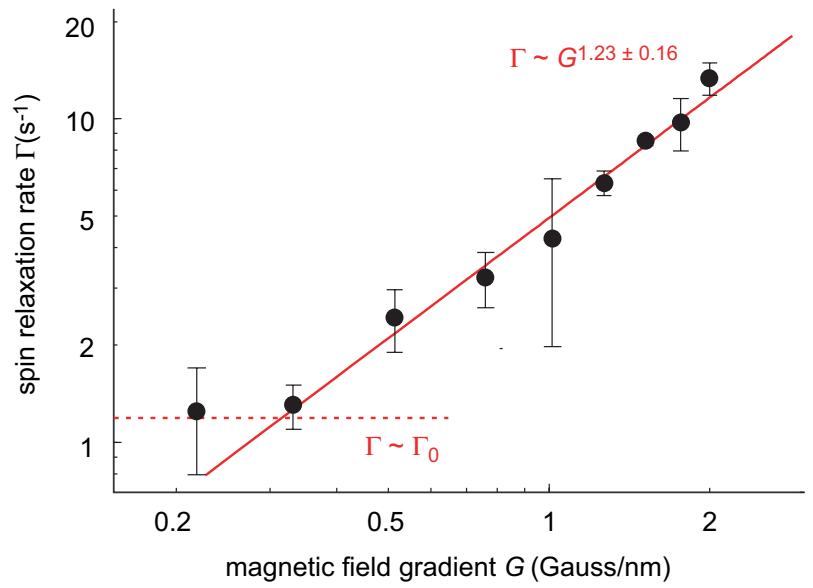

FIG. 11: Spin relaxation rate $\Gamma$ as a function of magnetic field gradient $G$. In the weak coupling regime, nuclear spin relaxation is dominated by interaction with lattice phonons $\left(\Gamma=\Gamma_{0}\right)$. In the strong coupling regime, spins relax via a specific low-frequency mechanical mode of the cantilever and $\Gamma \propto G^{-1.23}$. Figure adapted from Ref. ${ }^{81}$.

\section{G. Force detected nuclear double resonance}

Most recently, the IBM group exploited couplings between different spin species to enhance the 3D imaging capability of MRFM with the chemical selectivity intrinsic to magnetic resonance. They developed a method of nuclear double-resonance that allows the enhancement of the polarization fluctuation rate of one spin species by applying an rf field to the second spin species, resulting in suppression of the MRFM signal ${ }^{74}$. The physics behind this approach is analogous to HartmannHahn cross-polarization (CP) in NMR spectroscopy ${ }^{82}$, but involves statistical rather than Boltzmann polarization. The IBM group was inspired by previous work done with Boltzmann polarizations at the ETH in Zürich demonstrating $\mathrm{CP}$ as an efficient chemical contrast mechanism for micrometer-scale one-dimensional MRFM imaging ${ }^{52,53,83}$. In the IBM experiment, MRFM was used to measure the transfer between statistical polarizations of ${ }^{1} \mathrm{H}$ and ${ }^{13} \mathrm{C}$ spins in ${ }^{13} \mathrm{C}$-enriched stearic acid. The development of a cross-polarization technique for statistical ensembles adds an important tool for generating chemical contrast to the recently demonstrated technique of nanometer-scale MRI.

\section{FUTURE DEVELOPMENTS}

Since its invention and early experimental demonstration in the nineties ${ }^{16,30,31}$, the MRFM technique has progressed in its magnetic sensitivity from the equivalent of $10^{9}$ to presently about 100 proton magnetic moments (see Fig. 3). In order to eventually detect single nuclear spins and to image molecules at atomic resolution, the signalto-noise ratio of the measurement must still improve by 
two orders of magnitude. It is not clear if these advances can be achieved by incremental progress to the key components of the instrument, i.e. cantilever force transducers and nanoscale magnetic tips, or whether major shifts in instrumentation and methodology will be necessary. In the following we review some of the key issues and potential avenues for future developments.

\section{A. Magnetic tips}

The magnetic force on the cantilever can be enhanced by increasing the magnetic field gradient $G$. This can be achieved by making higher quality magnetic tips with sharp features and high-moment materials, and by simultaneously bringing the sample closer to these tips. To date, the highest magnetic field gradients have been reported in studies of magnetic disk drive heads, ranging between $20 \times 10^{6}$ and $40 \times 10^{6} \mathrm{~T} / \mathrm{m}^{84}$. The pole tips used in drive heads are typically made of soft, high-moment materials like FeCo, and have widths below $100 \mathrm{~nm}$. The magnetic tips used in the latest MRFM experiments, on the other hand, are more than $200 \mathrm{~nm}$ in diameter, and generate field gradients of less than $5 \times 10^{6} \mathrm{~T} / \mathrm{m}$. Moreover, calculations indicate that these tips do not achieve the ideal gradients which one would calculate assuming that they were made of pure magnetic material. This discrepancy may be due to a dead-layer on the outside of the tips, to defects inside the tips, or to contamination of the magnetic material. By improving the material properties and shrinking dimension of present MRFM tips, $G$ could be increased by up to a factor of ten. In practice, however, it will be difficult to gain an order of magnitude in signal-to-noise purely by improving the magnetic tips. To achieve higher gradients - and therefore higher signal-to-noise - we must resort to decreasing the tipsample spacing.

\section{B. Force noise near surfaces}

Since the gradient strength falls off rapidly with distance, bringing the sample closer to the magnetic tip would also increase the field gradient. However, measurements at small tip-sample spacings are hampered by strong tip-sample interactions which produce mechanical noise and dissipation in the cantilever. At the moment, imaging experiments are limited to spacings on the order of $25 \mathrm{~nm}$. For some experimental arrangements, surface dissipation can be observed at separations well over $100 \mathrm{~nm}$. This interaction has been studied in similar systems ${ }^{85,86}$ and several mechanisms have been proposed to explain its origin depending on the details of the configuration $^{87-91}$. Most explanations point to trapped charges or dielectric losses in either the substrate or the cantilever. Experimentally, several strategies could mitigate non-contact friction effects, including chemical modification of the surface, narrow tip size, or high-frequency operation. None of these approaches has yet emerged as the clear path for future improvement.

\section{Mechanical transducers}

The second means to improving the signal-to-noise ratio is the development of more sensitive mechanical transducers, i.e. transducers that exhibit a lower force noise spectral density $S_{F}$. For a mechanical resonator, $S_{F}$ is given by:

$$
S_{F}=\frac{4 k_{B} T m \omega_{0}}{Q},
$$

where $k_{B}$ is the Boltzmann constant, $T$ is the temperature, $m$ is the effective motional mass of the cantilever, $\omega_{0}$ is the angular resonance frequency of the cantilever's fundamental mode, and $Q$ the mechanical quality factor. In practice, this requires going to lower temperatures and making cantilevers which simultaneously have low $m \omega_{0}$ and large $Q$.

None of these steps is as straightforward as it first appears. Temperatures below $100 \mathrm{mK}$ can be achieved by cooling the apparatus in a dilution refrigerator. In the latest experiments, however, the cantilever temperature was limited to about $1 \mathrm{~K}$ by laser heating from the interferometer displacement sensor, not by the base temperature of the apparatus. Progress to sub-100 mK temperatures will therefore require new developments in displacement sensing.

The best strategies for maximizing $Q$ are not wellunderstood either. Apart from a loose trend that $Q$ often scales with thickness ${ }^{92}$, and a few general rules of thumb, i.e. minimizing clamping losses by design and keeping the mechanical resonator pristine and free of defects and impurities, no clear path has emerged. Holding $Q$ constant, one finds from simple Euler-Bernoulli beam theory that the product of $m$ and $\omega_{0}$ is minimized for cantilevers that are long and thin.

On a more fundamental level, it is worth considering the use of different materials and alternative geometries. Over the past few years a variety of nanomechanical resonators have been developed which rival the force sensitivities of the single crystal Si cantilevers used in most MRFM experiments. Some examples are the SiN membranes serving as sample stages in transmission electron microscopy ${ }^{93}$, vapor grown silicon nanowires ${ }^{94}$, and strained $\mathrm{SiN}$ or aluminum beams ${ }^{12,95}$. With some exceptions, the general trend is towards smaller resonators that more closely match the atomic lengthscales of spins and molecules. Therefore, it appears likely that future transducers will emerge as "bottom-up" structures rather than the "top-down" structures of the past. Instead of processing and etching out small mechanical devices out of larger bulk crystals, future resonators will probably be chemically grown or self-assembled: For example, they will be macroscale "molecules" such as nanowires, nanotubes ${ }^{96}$, or single sheets of graphene ${ }^{97}$. 
Although uncontrolled bottom-up approaches tend to be "dirty", remarkable mechanical properties can be achieved if care is taken to keep this self assembly process "clean". Most recently, researchers have demonstrated suspended carbon nanotubes with resonant frequencies of $250 \mathrm{MHz}$, masses of $10^{-20} \mathrm{~kg}$, and quality factors of $10^{598,99}$. If such a carbon nanotube force transducer could be operated at the thermal limit, which would require improved displacement detectors capable of measuring the nanotube's thermal motion, the resulting force sensitivity would be $0.01 \mathrm{aN} / \sqrt{\mathrm{Hz}}$, about 50 times better than any known mechanical force sensor today.

\section{Displacement sensors}

The mechanical deflection caused by spin or thermal force is typically a fraction of an Angstrom. In order to transfer the deflection into experimentally accessible electronic signals, very sensitive displacement sensor must be employed. To the best of our knowledge, all MRFM measurements have made use of optical detectors based on either optical beam deflection or laser interferometry. While optical methods provide an extremely sensitive means of measuring cantilever displacement, they face limitations as cantilevers become smaller and temperatures lower.

The first limitation comes about as the push for better spin sensitivity necessitates smaller and smaller cantilevers. The reflective areas of these levers will shrink to the order of, or even below, the wavelength of light. As a result, optical sensors will become less and less efficient as smaller and smaller fractions of the incident light are reflected back from the resonators. Thus, for the next generation of cantilevers - made from nanowires and nanotubes - interferometric displacement sensing may no longer be an option.

In principle, the inefficient reflection from small resonators can be balanced by increased laser power. Indeed, in a recent experiment, Nichol and coworkers have been able to sense the motion of $\mathrm{Si}$ nanowires at room temperature with diameters on the order of 20-nm using optical interferometry ${ }^{94}$. The researchers used a polarization resolved interferometer and a high incident laser power in order to sense the cantilever's motion.

High optical powers are, however, not compatible with low temperature operation. Especially at millikelvin temperatures, most materials (except for metals) have very poor thermal conductivities, and even very low incident laser powers can heat the cantilever. For example, a laser power of only $20 \mathrm{nW}$ from a $1550-\mathrm{nm}$ laser is sufficient to increase the temperature of a single crystal $\mathrm{Si}$ cantilever of the type shown in Fig. 3 from less than 100 $\mathrm{mK}$ to $300 \mathrm{mK}$, even though absorption is known to be minimal for this wavelength.

There are several potential displacement detectors which could achieve better sensitivity than optical methods while causing less measurement back-action, or heat- ing. An idea pursued by one of the authors is to make an off-board capacitively-coupled cantilever displacement detector based on a quantum point contact $(\mathrm{QPC})^{100}$. Preliminary measurements indicate that such a detector reaches at least the sensitivity of optical methods for equivalent cantilevers, with no indication of back-action from the electrons flowing in the device. While more work needs to be done, these kinds of capacitively coupled detectors are promising means of measuring mechanical resonators much smaller than the wavelength of light. One might imagine a future MRFM detection set-up where an arbitrarily small cantilever could be used, and a capacitive displacement detector is integrated on chip with a high-gradient magnetic tip, and an rf microwire source. Outstanding displacement sensitivities have also been achieved with microwave interferometers ${ }^{12,95}$, superconducting single-electron transistors, or high-finesse optical cavities made from micro-toroids which are very sensitive to fluctuations of nearby objects ${ }^{101}$. All of these latter displacement sensors will, however, need adjustments in order to be integrated in a contemporary scanning MRFM instrument.

\section{COMPARISON TO OTHER TECHNIQUES}

The unique position of MRFM among high-resolution microscopies becomes apparent when comparing it to other, more established nanoscale imaging techniques. As a genuine scanning probe method, MRFM has the potential to image matter at atomic resolution. While atomic-scale imaging is routinely achieved in scanning tunneling microscopy and atomic force microscopy, these techniques are confined to the top layer of atoms and cannot penetrate below surfaces ${ }^{102,103}$. Moreover, in standard scanning probe microscopy (SPM), it is difficult and in many situations impossible to identify the chemical species being imaged. Since MRFM combines SPM and MRI, these restrictions are lifted. The three-dimensional nature of MRI permits acquisition of sub-surface images with high spatial resolution even if the probe is relatively far away. As with other magnetic resonance techniques, MRFM comes with intrinsic elemental contrast and can draw from established NMR spectroscopy procedures to perform detailed chemical analysis. In addition, MRI does not cause any radiation damage to samples, as do electron and X-ray microscopies.

MRFM also distinguishes itself from super-resolution optical microscopies that rely on fluorescence imaging ${ }^{104}$. On the one side, optical methods have the advantage of working in vivo and they have the ability to selectively target the desired parts of a cell. Fluorescent labeling is now a mature technique which is routinely used for cellular imaging. On the other side, pushing the resolution into the nanometer range is hampered by fundamental limitations, in particular the high optical powers required and the stability of the fluorophores. Moreover, fluorescent labeling is inextricably linked with a modification of 
the target biomolecules, which alters the biofunctionality and limits imaging resolution to the physical size of the fluorophores.

MRFM occupies a unique position among other nanoscale spin detection approaches. While single electron spin detection in solids has been shown using several techniques, these mostly rely on the indirect read-out via electronic charge ${ }^{105,106}$ or optical transitions ${ }^{107,108}$. In another approach, the magnetic orientation of single atoms has been measured via the spin-polarized current of a magnetic STM tip or using magnetic exchange force microscopy ${ }^{109-111}$. These tools are very valuable to study single surface atoms, however, they are ill suited to map out sub-surface spins such as paramagnetic defects. In contrast, MRFM directly measures the magnetic moment of a spin, without resorting to other degrees of freedom, making it a very general method. This direct measurement of magnetic moment (or magnetic stray field) could also be envisioned using other techniques, namely SQuID microscopy ${ }^{112}$, Hall microscopy ${ }^{113}$, or recently introduced diamond magnetometry based on single nitrogen-vacancy centers $^{114-116}$. So far, however, none of these methods have reached the level of sensitivity needed to detect single electron spins, or volumes of nuclear spins much less than one micrometer ${ }^{117,118}$. It is certainly possible that future improvements to these methods - especially to diamond magnetometry - may result in alternative techniques for nanoscale MRI that surpass the capabilities of MRFM.

\section{OUTLOOK}

Despite the tremendous improvements made to MRFM over the last decade, several important obstacles must be overcome in order to turn the technique into a useful tool for biologists and materials scientists. Most existing MRFM instruments are technically involved prototypes; major hardware simplifications will be required for routine screening of nanoscale samples. Suitable specimen preparation methods must be developed that are compatible with the low temperature, high vacuum environment required for the microscope to operate at its highest sensitivity and resolution. While this is particularly challenging for biological samples, protocols exist which could be adapted to MRFM. In cryo-electron microscopy, for example, dispersed samples are vitrified to preserve their native structure by plunge-freezing in liquid nitrogen ${ }^{119}$. As objects become smaller, isolation of samples and suppression of unwanted background signals from surrounding material will become increasingly important.

The conditions under which the latest MRFM imaging experiments were carried out are remarkably similar to those prevailing in cryo-electron microscopy, the highest resolution 3D imaging technique commonly used by structural biologists today. Cryo-electron microscopy, like MRFM, operates at low temperatures and in high vacuum, requires long averaging times (on the order of days) to achieve sufficient contrast, and routinely achieves resolutions of a few nanometers ${ }^{120,121}$. Unlike MRFM, however, electron microscopy suffers from fundamental limitations that severely restrict its applicability. Specimen damage by the high-energy electron radiation limits resolution to 5-10 nm if only a single copy of an object is available. Averaging over hundreds to thousands of copies is needed to achieve resolutions approaching 10 $\AA^{122}$. In addition, unstained images have intrinsically low contrast, whereas staining comes at the expense of modifying the native structure.

MRFM has the unique capability to image nanoscale objects in a non-invasive manner and to do so with intrinsic chemical selectivity. For this reason the technique has the potential to extend microscopy to the large class of structures that show disorder and therefore cannot be averaged over many copies. These structures include such prominent examples as HIV, Influenza virus, and Amyloid fibrils. Virtually all of these complexes are associated with important biological functions ranging from a variety of diseases to the most basic tasks within the cellular machinery. For such complexes, MRFM has the potential not only to image the three-dimensional macromolecular arrangement, but also to selectively image specific domains in the interior through isotopic labeling.

While the most exciting prospect for MRFM remains its application to structural imaging in molecular biology, its applications are not limited to biological matter. For example, most semiconductors contain non-zero nuclear magnetic moments. Therefore MRFM may prove useful for sub-surface imaging of nanoscale electronic devices. MRFM also appears to be the only technique capable of directly measuring the dynamics of the small ensembles of nuclear spin that limit electron spin coherence in single semiconductor quantum dots. Polymer films and self-assembled monolayers - important for future molecular electronics - are another exciting target for MRFM and its capability to image chemical composition on the nanoscale. Finally, isotopically engineered materials are becoming increasingly important for tuning a variety of physical properties such as transport and spin. Researchers currently lack a general method for non-invasively imaging the isotopic composition of these materials ${ }^{123-125}$; MRFM techniques could fill this void.

As force-detected magnetic resonance has traditionally been an exploratory field, it is possible that applications other than nanoscale imaging will emerge. Single electron spin detection, for example, is an important prerequisite for future quantum information applications ${ }^{7,126}$. At the same time, MRFM may also become an important tool in the study of defects or dopants deep in materials, or for mapping of spin labels in decorated biological nanostructures ${ }^{127}$. The key components to the instrument - in particular the ultrasensitive micromechanical cantilevers, nanomagnetic tips, and displacement transducers - could also find new applications outside the area of spin detection. 


\section{CONCLUSION}

Over the last two decades, MRFM has led to exciting progress in the field of ultrasensitive spin detection and high-resolution MRI microscopy. Starting with early demonstrations in the 1990s imaging with resolutions of a few micrometers - on par with conventional MRI microscopy - the technique has progressed to the point where it can resolve single virus particles and molecular monolayers. Given the fast pace at which modern nanofabrication technology is evolving, an improvement of the method down to one-nanometer resolution seems feasible without major changes to the instrument. This resolution, which is comparable to what three-dimensional electron microscopy reaches on biological specimens, would be sufficient to map out the coarse structure of many macromolecular complexes. The extension of MRFM to atomic resolution, where atoms in molecules could be directly mapped out and located in $3 \mathrm{D}$, remains an exciting if technically very challenging prospect.

\section{Acknowledgments}

The work discussed in this review was only possible because of the many fruitful experimental collaborations with H. J. Mamin and D. Rugar of the IBM Almaden Research Center. The authors also thank these colleagues for their many detailed comments and very helpful discussions pertaining to this review.
1 K. C. Schwab and M. L. Roukes, Phys. Today 58, No. 7, 36 (2005).

2 A. N. Cleland, Foundations of Nanomechanics (SpringerVerlag, Berlin, 2003).

${ }^{3}$ K. L. Ekinci and M. L. Roukes, Rev. Sci. Instrum. 76, 061101 (2005).

4 M. Blencowe, Phys. Rep. 395, 159 (2004).

${ }^{5}$ H. B. Chan et al. Science 291, 1941 (2001); M. Bordag, U. Mohideen, and V. M. Mostepanenko, Phys. Rep. 353 , 1 (2001).

6 S. J. Smullin et al. Phys. Rev. D 72, 122001 (2005).

7 D. Rugar et al. Nature 430, 329 (2004).

8 A. C. Bleszynski-Jayich et al. Science 326, 272-275 (2009).

9 T. D. Stowe et al. Appl. Phys. Lett. 71, 288 (1997).

10 H. J. Mamin and D. Rugar, Appl. Phys. Lett. 79, 3358 (2001).

11 A. Naik et al. Nature 443, 193-196 (2006).

12 J. D. Teufel et al. Nature Nanotech. 4, 820-823 (2009).

13 R. G. Knobel and A. N. Cleland, Nature 424, 291 (2003).

14 M. D. LaHaye et al. Science 304, 74 (2004).

15 T. Rocheleau et al. Nature doi:10.1038/nature08681 (2009).

16 J. A. Sidles, Appl. Phys. Lett. 58, 2854 (1991).

17 J. A. Sidles, Phys. Rev. Lett. 68, 1124 (1992).

18 J. A. Sidles, J. L. Garbini, and G. P. Drobny, Rev. Sci. Instrum. 63, 3881 (1992).

19 C. L. Degen, M. Poggio, H. J. Mamin, C. T. Rettner, and D. Rugar, Proc. Nat. Acad. Sci. U.S.A. 106, 1313 (2009).

20 J. A. Sidles et al. Rev. Mod. Phys. 67, 249 (1995).

21 N. Nestle, A. Schaff, and W. S. Veeman, Progress in $\mathrm{Nu}$ clear Magnetic Resonance Spectroscopy 38, 1 (2001).

22 A. Suter, Progress in Nuclear Magnetic Resonance Spectroscopy 45, 239 (2004).

${ }^{23}$ G. P. Berman, F. Borgonovi, V. N. Gorshkov, and V. I. Tsifrinovich (authors), Magnetic Resonance Force Microscopy And a Single-spin Measurement, (World Scientific Publishing Company, 2006).

24 "The Magnetic Resonance Microscope," P. C. Hammel and D. V. Pelekhov, Book Chapter, Handbook of Magnetism and Advanced Magnetic Materials, H. Kronmüller and S. Parkin, eds., Volume 5: Spintronics and Magneto- electronics, Wiley, ISBN: 978-0-470-02217-7.

25 S. Kuehn, S. A. Hickman, and J. A. Marohn, J. Chem. Phys. 128052208 (2008).

26 M. Barbic, Magnetic Resonance Microscopy, edited by S. L. Codd and J. D. Seymour, (Wiley, 2009), p. 49-63.

27 L. Ciobanu, D. A. Seeber, and C. H. Pennington, J. Magn. Reson. 158, 178 (2002); S.-C. Lee et al., J. Magn. Reson. 150, 207 (2001); P. Glover and P. Mansfield, Rep. Prog. Phys. 65, 1489 (2002).

28 D. F. Evans, Philosophical Magazine 1, 370 (1956).

29 G. Alzetta, E. Arimondo, C. Ascoli, and A. Gozzini, Il Nuovo Cimento B 62, 392 (1967).

30 D. Rugar, C. S. Yannoni, and J. A. Sidles, Nature 360, 563 (1992).

31 D. Rugar et al. Science 264, 1560 (1994).

32 Z. Zhang, P. C. Hammel, P. E. Wigen, Appl. Phys. Lett. 68, 2005 (1996).

33 K. Wago, O. Züger, R. Kendrick, C. S. Yannoni, and D. Rugar, J. Vac. Sci. Technol. B 14, 1197 (1996).

${ }^{34}$ K. Wago, D. Botkin, C. S. Yannoni, and D. Rugar, Appl. Phys. Lett. 72, 2757 (1998).

35 K. J. Bruland, W. M. Dougherty, J. L. Garbini, J. A. Sidles, and S. H. Chao, Appl. Phys. Lett. 73, 3159 (1998).

36 B. C. Stipe, H. J. Mamin, C. S. Yannoni, T. D. Stowe, T. W. Kenny, and D. Rugar, Phys. Rev. Lett. 87, 277602 (2001).

37 S. R. Garner, S. Kuehn, J. M. Dawlaty, N. E. Jenkins, and J. A. Marohn, Appl. Phys. Lett. 84, 5091 (2004).

38 H. J. Mamin, R. Budakian, B. W. Chui, and D. Rugar, Phys. Rev. Lett. 91, 207604 (2003).

39 O. Züger and D. Rugar, Appl. Phys. Lett. 63, 2496 (1993).

40 O. Züger and D. Rugar, J. Appl. Phys. 75, 6211 (1994).

41 O. Zuger, S. T. Hoen, C. S. Yannoni, and D. Rugar, J. Appl. Phys. 79, 1881 (1996).

42 A. Blank, C. R. Dunnam, P. P. Borbat, and J. H. Freed, J. Magn. Res. 165, 116 (2003).

${ }^{43}$ K. R. Thurber, L. E. Harrell, and D. D. Smith, J. Magn. Res. 162, 336 (2003).

44 S. Tsuji, T. Masumizu, and Y. Yoshinari, J. Magn. Reson. 167, 211-220 (2004).

45 S. Chao, W. M. Dougherty, J. L. Garbini, and J. A. Sidles, Rev. Sci. Instr. 75, 1175 (2004). 
${ }^{46}$ K. Wago, O. Züger, J. Wegener, R. Kendrick, C.S . Yannoni, and D. Rugar, Rev. Sci. Instr. 68, 1823 (1997).

47 A. Schaff and W. S. Veeman, J. Magn. Res. 126, 200 (1997).

48 K. Wago, D. Botkin, C. S. Yannoni, and D. Rugar, Phys. Rev. B 57, 1108 (1998).

49 R. Verhagen et al. J. Am. Chem. Soc. 124, 1588 (2002).

50 C. L. Degen et al. Phys. Rev. Lett. 94, 207601 (2005).

51 Q. Lin, C. L. Degen, M. Tomaselli, A. Hunkeler, U. Meier, and B. H. Meier, Phys. Rev. Lett. 96, 137604 (2006).

52 K. W. Eberhardt, Q. Lin, U. Meier, A. Hunkeler, and B. H. Meier, Phys. Rev. B 75, 184430 (2007).

${ }^{53}$ K. W. Eberhardt, C. L. Degen, A. Hunkeler, and B. H. Meier, Angew. Chem. Int. Ed. 47, 8961 (2008).

${ }^{54}$ C. Ascoli, P. Baschieri, C. Frediani, L. Lenci, M. Martinelli, G. Alzetta, R. M. Celli, and L. Pardi, Appl. Phys. Lett. 69, 3920 (1996).

55 G. M. Leskowitz, L. A. Madsen, and D. P. Weitekamp, Solid State Nuclear Magnetic Resonance 11, 73 (1998).

56 L. A. Madsen et al. Proc. Natl. Acad. Sci. USA 101, 12804 (2004).

57 P. Wigen, M. Roukes, and P. Hammel, Ferromagnetic resonance force microscopy, in: Topics in Applied Physics Spin Dynamics in Confined Magnetic Structures III, Vol. 101, (Springer, Berlin, 2006), p. 105-136.

58 G. de Loubens, V. V. Naletov, O. Klein, J. B. Youssef, F. Boust, and N. Vukadinovic, Phys. Rev. Lett. 98, 127601 (2007).

59 B. W. Chui et al. TRANSDUCERS, 12th International Conference on Solid-State Sensors, Actuators and $\mathrm{Mi}$ crosystems 2, 1120 (2003).

60 B. C. Stipe et al. Phys. Rev. Lett. 86, 2874 (2001).

61 R. Budakian, H. J. Mamin, B. W. Chui, D. Rugar, Science 307, 408 (2005).

62 H. J. Mamin, M. Poggio, C. L. Degen, and D. Rugar, Nature Nanotech. 2, 301 (2007).

63 M. Poggio et al. Appl. Phys. Lett. 90, 263111 (2007).

64 N. Dobigeon, A. O. Hero, J.-Y. Tourneret, IEEE Trans. Image Process. 18, 2059 (2009).

65 H. J. Mamin et al. Nano Lett. 9, 3020 (2009).

66 F. Bloch, Phys. Rev. 70, 460 (1946).

67 T. Sleator, E. L. Hahn, C. Hilbert and J. Clarke, Phys. Rev. Lett. 55, 1742 (1985).

68 M. A. McCoy, and R. R. Ernst, Chem. Phys. Lett. 159, 587 (1989).

69 M. Guéron, and J. L. Leroy, J. Magn. Reson. 85, 209 (1989).

70 N. Müller, and A. Jerschow, Proc. Natl. Ac. Sci. 103, 6790 (2006).

71 S. A. Crooker, D. G. Rickel, A. V. Balatsky and D. L. Smith, Nature 431, 49 (2004).

72 H. J. Mamin, R. Budakian, B. W. Chui, and D. Rugar, Phys. Rev. B 72, 024413 (2005).

73 C. L. Degen, M. Poggio, H. J. Mamin, and D. Rugar, Phys. Rev. Lett. 99, 250601 (2007).

74 M. Poggio, H. J. Mamin, C. L. Degen, M. H. Sherwood, and D. Rugar, Phys. Rev. Lett. 102, 087604 (2009).

75 D. Mozyrsky, I. Martin, D Pelekhov, and P. C. Hammel, App. Phys. Lett. 82, 1278 (2003).

76 G. P. Berman, V. N. Gorshkov, D. Rugar, and V. I. Tsifrinovich, Phys. Rev. B 68, 094402 (2003).

77 P. C. M. M. Magusin, and W. S. Veeman, J. Magn. Res. 143, 243 (2000).

78 M. C. Butler, V. A. Norton, and D. P. Weitekamp, Ab- stracts of papers of the American Chemical Society 229, U735-U735 217-PHYS, 2005; D. P. Weitekamp, Patent US 6,841,995 B2, 2005.

79 I. Bargatin and M. Roukes, Phys. Rev. Lett 91, 138302 (2003).

${ }^{80}$ P. Rabl, S. J. Kolkowitz, F. H. Koppens, J. G. E. Harris, P. Zoller, M. D. Lukin, arXiv:0908.0316.

81 C. L. Degen, M. Poggio, H. J. Mamin, and D. Rugar, Phys. Rev. Lett. 100, 137601 (2008).

82 S. R. Hartmann and E. L. Hahn, Phys. Rev. 128, 2042 (1962).

83 Q. Lin et al. Phys. Rev. Lett. 96, 137604 (2006).

${ }^{84}$ C. Tsang et al., IEEE Trans. Magn. 42, 145 (2006).

85 B. C. Stipe, H. J. Mamin, T. D. Stowe, T. W. Kenny, and D. Rugar, Phys. Rev. Lett. 87, 096801 (2001).

86 S. Keuhn, R. F. Loring, J. A. Marohn, Phys. Rev. Lett. 96, 156103 (2006).

87 B. N. J. Persson and Z. Zhang, Phys. Rev. B 57, 7327 (1998).

88 J. R. Zurita-Sanchez, J. J. Greffet, and L. Novotny, Phys. Rev. A 69, 022902 (2004).

89 A. I. Volokitin and B. N. J. Persson, Phys. Rev. Lett. 91, 106101 (2003).

90 A. I. Volokitin and B. N. J. Persson, Phys. Rev. Lett. 94, 086104 (2005).

91 J. Labaziewicz, Y. Ge, D. R. Leibrandt, S. X. Wang, R. Shewmon, and I. L. Chuang, Phys. Rev. Lett. 101, 180602 (2008).

92 K. Y. Yasumura et al. J. Microelectromech. Sys. 9, 117 (2000).

93 J. D. Thompson et al. Nature 452, 72 (2008).

94 J. M. Nichol et al. Appl. Phys. Lett. 93, 193110 (2008).

95 T. Rocheleau, T. Ndukum, C. Macklin, J. B. Hertzberg, A. A. Clerk, and K. C. Schwab, Nature 463, 72 (2010).

96 V. Sazonova et al. Nature 431, 284 (2004).

97 J. S. Bunch et al. Science 315, 490 (2007).

98 A. K. Huttel et al. Nano Lett. 9, 2547 (2009).

99 G. A. Steele et al. Science 325, 1103 (2009).

100 M. Poggio et al. Nature Phys. 4, 635 (2008).

101 G. Anetsberger et al. Nature Phys. 5, 909 (2009).

102 P. Hansma, and J. Tersoff, J. Appl. Phys. 61, R1-R23 (1987).

103 F. Giessibl, Rev. Mod. Phys. 75, 949-983 (2003).

104 B. Huang, M. Bates, and X. Zhuang, Annu. Rev. Biochem. 78, 993-1016 (2009).

105 J. M. Elzerman et al., Nature 430, 431 (2004).

106 M. Xiao, I. Martin, E. Yablonovitch, and H. W. Jiang, Nature 430, 435 (2004).

107 J. Wrachtrup, C. von Borczyskowski, J. Bernard, M. Orritt, and R. Brown, Nature 363, 244 (1993).

108 F. Jelezko, I. Popa, A. Gruber, C. Tietz, and J. Wrachtrup, Appl. Phys. Lett. 81, 2160 (2002).

109 C. Durkan, and M. E. Welland, Appl. Phys. Lett. 80, 458 (2002).

110 S. Heinze et al., Science 288, 1805-1808 (2000).

111 U. Kaiser, A. Schwarz, and R. Wiesendanger, Nature 446, 522-525 (2007).

112 J. R. Kirtley et al., Appl. Phys. Lett. 66, 1138 (1995).

113 A. M. Chang et al., Appl. Phys. Lett. 61, 1974 (1992).

114 C. L. Degen, Appl. Phys. Lett. 92, 243111 (2008).

115 J. R. Maze et al., Nature 455, 644 (2008).

116 G. Balasubramanian et al., Nature 455, 648 (2008).

117 C. L. Degen, Nature Nanotech. 3, 643 (2008).

118 A. Blank, E. Suhovoy, R. Halevy, L. Shtirberg, and W. 
Harneit, Phys. Chem. Chem. Phys. 11, 6689-6699 (2009).

119 K. A. Taylor, and R. M. Glaeser, Science 186, 1036 (1974).

120 V. Lucic, F. Forster, and W. Baumeister, Annual Rev. of Biochemistry 74, 833 (2005).

121 S. Subramaniam, Current Opinion in Microbiology 8, 316 (2005).

122 R. M. Glaeser, Physics Today 61, 48 (2008).

123 Y. Shimizu, and K. Itoh, Thin Solid Films 508, 160-162 (2006).
124 I. Shlimak, V. Safarov, and I. Vagner, J. Phys. Cond. Mat. 13, 6059 (2001).

125 T. Kelly, and M. Miller, Rev. Sci. Instrum. 78, 031101 (2007).

126 B. Kane, Fortschritte der Physik 48, 1023-1041 (2000).

127 E. W. Moore, S. Lee, S. A. Hickman, S. J. Wright, L. E. Harrell, P. P. Borbat, J. H. Freed, and J. A. Marohn, Proc. Nat. Acad. Sci. U.S.A. 106, 22251-22256 (2009). 